\title{
QUANTIDADE RELATIVA DE FÓSFORO FIXADO EM UM SOLO HIDROMÓRFICO *
}

\author{
Francisco José de Albuquerque Cavalcanti** \\ Francisco de Assis Ferraz de Mello***
}

\section{RESUMO}

Utilizando um solo Hidromórfico, Série Três Municípios, que ocorre na região de Piracicaba, São Paulo, fez-se um estudo sobre o efeito do período de incubação e do teor de fósforo de uma solução adicionada ao solo na quantidade de fósforo fixado.

A quantidade relativa de fósforo fixado foi obtida mediante um esquema previamente estabelecido, a partir de 4 períodos de incubação $(0-2-20$ e 200 horas $)$ e de 4 concentraçôes da solução de fosfato monocálcito $(0-500-1.000$ e $1.500 \mathrm{ppm}$ de P).

Cada tratamento constou de uma quantidade de amostra equivalente a $10 \mathrm{~g}$ de TFSE que recebeu $4 \mathrm{ml}$ de uma solução de concentração conhecida. Findo o período de incubação para cada caso, determinou-se o teor de fósforo no extrato obtido com uma solução $0,025 \mathrm{~N}$ em $\mathrm{H}_{2} \mathrm{SO}_{4}$ e $0,05 \mathrm{~N}$ em $\mathrm{HCl}$.

Os resultados encontrados revelaram que a quantidade relativa de fósforo fixado, de modo como foi obtida, prestou-se bem para as finalidades deste trabalho. A análise estatística desses dados mostrou que os fatores estudados não atuaram isoladamente e que com cerca de $60 \%$ do maior perído de incubação seria alcançada a quantidade máxima de fósforo fixado.

\section{INTRODUÇÃO}

De acordo com a natureza de seus constituintes e dos fatores envolvidos no processo, cada solo apresenta uma determinada capacidade para fixar o fósforo. Por sua vez, a quantidade de fósforo retida por um determinado solo sofrerá alteração à medida que se modifica a ação de um ou mais fatores. O problema torna-se ainda mais complexo quando esses fatores interferem também na absorção do fósforo pelas plantas como é o caso, por exemplo, da textura e teor da umidade (MAHTAB et al., 1971).

* Trabalho realizado com parte dos dados contidos na Dissertação apresentada pelo primeiro autor à E.S.A. "Luiz de Queiroz" da Universidade de São Paulo (ESALQ-USP em 1974). Entregue para publicação em 27-12-77.

* Eng. ${ }^{\circ}$ Agr.o, Mestre em Solos e Nutrição de Plantas, da Seção de Solos do Instituto de Pesquisa Agropecuária do Nordeste (IPEANE), Recife, Pernambuco.

*** Professor Adjunto do Departamento de Solos, Geologia e Fertilizantes da ESALQ. 
Por outro lado, a um solo que recebeu quantidade de fósforo superior à necessidade para atingir a sua capacidade de saturação, pode-se aplicar mais desse elemento, numa forma solúvel, sem se correr o risco dele vir a ser fixado (BASU \& MUCHERJES, 1972).

Considerando a possibilidade de se atingir a capacidade de saturação e tendo-se em vista ainda que o equilíbrio do sistema solo-solução fosfatada só será atingido após um certo período de tempo, procurou-se estudar a ação conjunta do período de incubação e da concentração de uma solução de fosfato monocálcico na quantidade de fósforo fixada. por um solo Hidromórfico.

Análise mecânica (\%):

$\begin{array}{lr}\text { Areia muito grossa }(2-1 \mathrm{~mm}) & 0,3 \\ \text { Areia grossa }(1-0,5 \mathrm{~mm}) & 2,6 \\ \text { Areia média }(0,5-0,25 \mathrm{~mm}) & 7,0 \\ \text { Areia fina }(0,25-0,10 \mathrm{~mm}) & 10,2 \\ \text { Areia muito fina }(0,10-0,05 \mathrm{~mm}) & 4,6 \\ \text { Limo }(0,05-0,002 \mathrm{~mm}) & 28,1 \\ \text { Argila }(<0,002 \mathrm{~mm}) & 47,2 \\ \text { Classe textural } & \text { Argiloso }\end{array}$

Análise química:

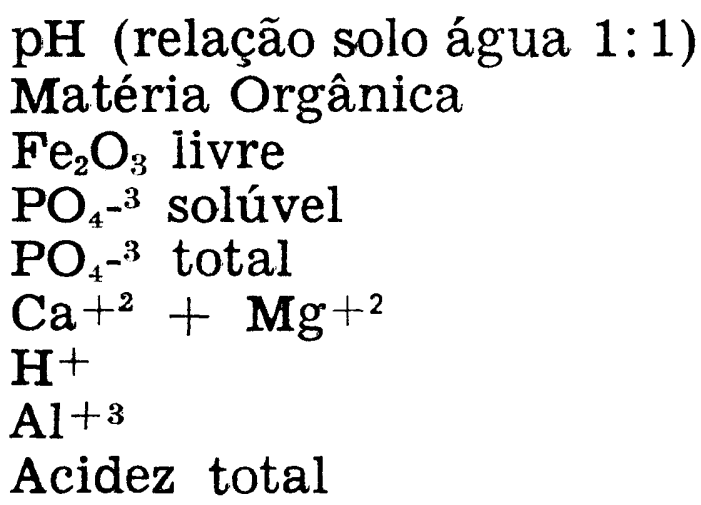

$\begin{array}{cl}\% & 5,2 \\ \% & 3,13 \\ \text { (e } \mathrm{mg} / 100 \mathrm{~g}) & 0,05 \\ \text { (e } \mathrm{mg} / 100 \mathrm{~g}) & 0,64 \\ \text { (e } \mathrm{mg} / 100 \mathrm{~g}) & 3,87 \\ \text { (e } \mathrm{mg} / 100 \mathrm{~g}) & 0,25 \\ \text { (e } \mathrm{mg} / 100 \mathrm{~g}) & 0,77 \\ \text { (e } \mathrm{mg} / 100 \mathrm{~g}) & 5,68\end{array}$

Serviu de base para o estudo sobre a influência do tempo de contacto e da concentração da solução na quantidade de fósforo fixado, o trabalho de WAUGH \& FITS (1966). Inicialmente foram estabelecidos 4 períodos de incubação $\left(\mathrm{T}_{0}=0, \mathrm{~T}_{1}=2, \mathrm{~T}_{2}=20 \mathrm{e} \mathrm{T}_{3}=200\right.$ horas $) \mathrm{e}$ 4 concentrações para a solução empregada $\left(\mathrm{P}_{0}=0, \mathrm{P}_{1}=500, \mathrm{P}_{2}=1.000\right.$ e $\mathrm{P}_{3}=1.500 \mathrm{ppm}$ de $\mathrm{P}$ ), sendo o número de repetições igual a 4 conforme o esquema da Fig. 1 . 


\begin{tabular}{|c|c|c|c|}
\hline $\mathrm{t}_{0} \mathrm{p}_{0}$ & $\mathrm{t}_{0} \mathrm{p}_{1}$ & $\mathrm{t}_{0} \mathrm{p}_{2}$ & $\mathrm{t}_{0} \mathrm{p}_{3}$ \\
\hline $\mathrm{t}_{1} \mathrm{p}_{0}$ & $\mathrm{t}_{1} \mathrm{p}_{1}$ & $t_{1} p_{2}$ & $\mathrm{t}_{1} \mathrm{p}_{3}$ \\
\hline $\mathrm{t}_{2} \mathrm{p}_{0}$ & $\mathrm{t}_{2} \mathrm{p}_{1}$ & $\mathrm{t}_{2} \mathrm{p}_{2}$ & $\mathrm{t}_{2} \mathrm{p}_{3}$ \\
\hline$t_{3} p_{0}$ & $t_{3} p_{1}$ & $\mathrm{t}_{3} \mathrm{p}_{2}$ & $\mathrm{t}_{3} \mathrm{p}_{3}$ \\
\hline
\end{tabular}

FIGURA 1 - Esquema dos tratamentos utilizados

\begin{tabular}{|c|c|c|c|}
\hline- & 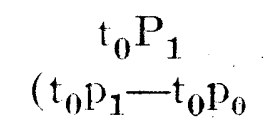 & $\left(\begin{array}{c}\mathrm{t}_{0} \mathrm{p}_{2} \\
\left(\mathrm{t}_{0} \mathrm{p}_{2}-\mathrm{t}_{0} \mathrm{p}_{0}\right)\end{array}\right.$ & $\left(\begin{array}{c}\mathrm{t}_{0} \mathrm{p}_{3} \\
\left(\mathrm{t}_{0} \mathrm{p}_{3}-\mathrm{t}_{0} \mathrm{p}_{0}\right)\end{array}\right.$ \\
\hline - & $\begin{array}{c}t_{1} p_{1} \\
\left(t_{1} p_{1}-t_{1} p_{0}\right)\end{array}$ & $\begin{array}{c}t_{1} p_{2} \\
\left(t_{1} p_{2}-t_{1} p_{0}\right)\end{array}$ & $\left(\begin{array}{c}\mathrm{t}_{1} \mathrm{p}_{3} \\
\left(\mathrm{t}_{1} \mathrm{p}_{3}-\mathrm{t}_{1} \mathrm{p}_{0}\right)\end{array}\right.$ \\
\hline- & $\left(t_{2} p_{1}-t_{2} p_{0}\right)$ & $\begin{array}{c}\left.t_{2} p_{2}\right) \\
\left(t_{2} p_{2}-t_{2} p_{0}\right)\end{array}$ & $\left(t_{2} p_{3}-t_{2} p_{20} p_{0}\right)$ \\
\hline- & $\begin{array}{c}{ }^{t_{3} p_{1}} \\
\left(t_{3} p_{1}-t_{3} p_{0}\right)\end{array}$ & $\left(\begin{array}{c}t_{3} p_{2} \\
\left(t_{3} p_{2}-t_{3} p_{0}\right)\end{array}\right.$ & $\begin{array}{c}\mathrm{t}_{3} \mathrm{p}_{3} \\
\left.\mathrm{t}_{3} \mathrm{p}_{3}-\mathrm{t}_{3} \mathrm{p}_{9}\right)\end{array}$ \\
\hline
\end{tabular}

FIGURA 2 - Tratamentos corrigidos de $\mathrm{P}_{0}$

A quantidade relativa de fósforo fixado foi obtida deduzindo-se inicialmente dos resultados de cada um dos tratamentos aqueles em que a solução não continha fósforo (Fig. 2). A seguir, os novos resultados foram subtraídos daqueles em que o tempo de contacto foi nulo (Fig. 3).

Os tratamentos tomaram uma forma fatorial $3 \times 3$ sendo escolhido para delineamento experimental o Inteiramente Casualizado, com 4 repetições.

\begin{tabular}{cccc}
\hline- & - & - & - \\
- & $\mathrm{T}_{1} \mathrm{P}_{1}$ & $\mathrm{~T}_{1} \mathrm{P}_{22}$ & $\mathrm{~T}_{1} \mathrm{P}_{2}$ \\
- & $\left(\mathrm{t}_{0} \mathrm{P}_{1}-\mathrm{t}_{1} \mathrm{P}_{1}\right.$ & $\left(\mathrm{t}_{0} \mathrm{P}_{2}-\mathrm{t}_{1} \mathrm{P}_{2}\right)$ & $\left(\mathrm{t}_{0} \mathrm{P}_{3}-\mathrm{t}_{1} \mathrm{P}_{3}\right)$ \\
- & $\mathrm{T}_{2} \mathrm{P}_{1}$ & $\mathrm{~T}_{2} \mathrm{P}_{2}$ & $\mathrm{~T}_{2} \mathrm{P}_{3}$ \\
- & $\left(\mathrm{t}_{0} \mathrm{P}_{1}-\mathrm{t}_{2} \mathrm{P}_{1}\right)$ & $\left(\mathrm{t}_{0} \mathrm{P}_{2}-\mathrm{t}_{2} \mathrm{P}_{22}\right)$ & $\left(\mathrm{t}_{0} \mathrm{P}_{3}-\mathrm{t}_{2} \mathrm{P}_{3}\right)$ \\
& $\left(\mathrm{T}_{3} \mathrm{P}_{1}\right.$ & $\mathrm{T}_{3} \mathrm{P}_{2}$ & $\ldots$ \\
& $\left(\mathrm{t}_{0} \mathrm{P}_{1}-\mathrm{t}_{3} \mathrm{P}_{1}\right.$ & $\left(\mathrm{t}_{0} \mathrm{P}_{2}-\mathrm{t}_{3} \mathrm{P}_{2}\right)$ & $\left(\mathrm{t}_{0} \mathrm{P}_{3}-\mathrm{t}_{3} \mathrm{P}_{3}\right)$ \\
\hline
\end{tabular}

FIGURA 3 - Esquema para obter-se as quantidades relativas de $\mathbf{P}$ fixadas 
Foi tomada para cada tratamento uma quantidade de amostra equivaliente a $10 \mathrm{~g}$ de TFSE onde adcionou-se $4 \mathrm{ml}$ de uma solução de concentração conhecida. A reposição da água perdida baseou-se no peso de cada unidade experimental.

Para a determinação do teor de fósforo solúvel ao término do ensaio recorreu-se ao método descrito por VETTORI (1966), tendo-se o cuidado de observar sempre um intervalo de aproximadamente 24 horas entre a extração e o restante da análise.

Os dados obtidos foram submetidos a uma análise estatística onde foram pesquisados os efeitos linear e quadrático, tanto do tempo de contacto ( $\mathrm{T}$ ) em $\mathrm{P}$, como da concentração da solução (P) em T. Por último, foi calculada a concentração e o tempo de contacto capaz de proporcionar a quantidade relativa máxima de fósforo fixado para o solo utilizado, nas condições deste trabalho.

\section{RESULTADOS E DISCUSSÃO}

O teor de fósforo nos extratos obtidus com uma solução $0,025 \mathrm{~N}$ em $\mathrm{H}_{2} \mathrm{SO}_{4}$ e $0,05 \mathrm{~N}$ em HCl, antes e depois de ser corrigido de $\mathrm{p}_{0}$, bem como a quantidade relativa de fósforo fixado, podem ser vistos no Quadro 1 .

A análise estatística dos resultados obtidos revelou uma significância estatística para tratamentos ao nível de $1 \%$, possibilitando a decomposição dos graus de liberdade e a pesquisa dos efeitos linear e quadrático, tanto de $\mathrm{P}$ dentro de $\mathrm{T}$, como de $\mathrm{T}$ dentro de $\mathrm{P}$ (Quadro 2 ).

Dessa forma, ficou constatado que apenas o efeito quadrático de $\mathrm{P}$ em $\mathrm{T}_{3}$ não foi significativo ao nível de $5 \%$.

Através das equações de regressão obtidas (Quadro 3) pôde-se constatar que elas não admitiram um máximo para os efeitos quadráticos de $\mathrm{P}$ em $\mathrm{T}$. Contudo a quantidade relativa máxima de fósforo dentro de cada valor de $P$ foi obtida com uma porcentagem inferior a $65 \%$ do maior período de incubação $\left(\mathrm{T}_{3}=200\right.$ horas) . 


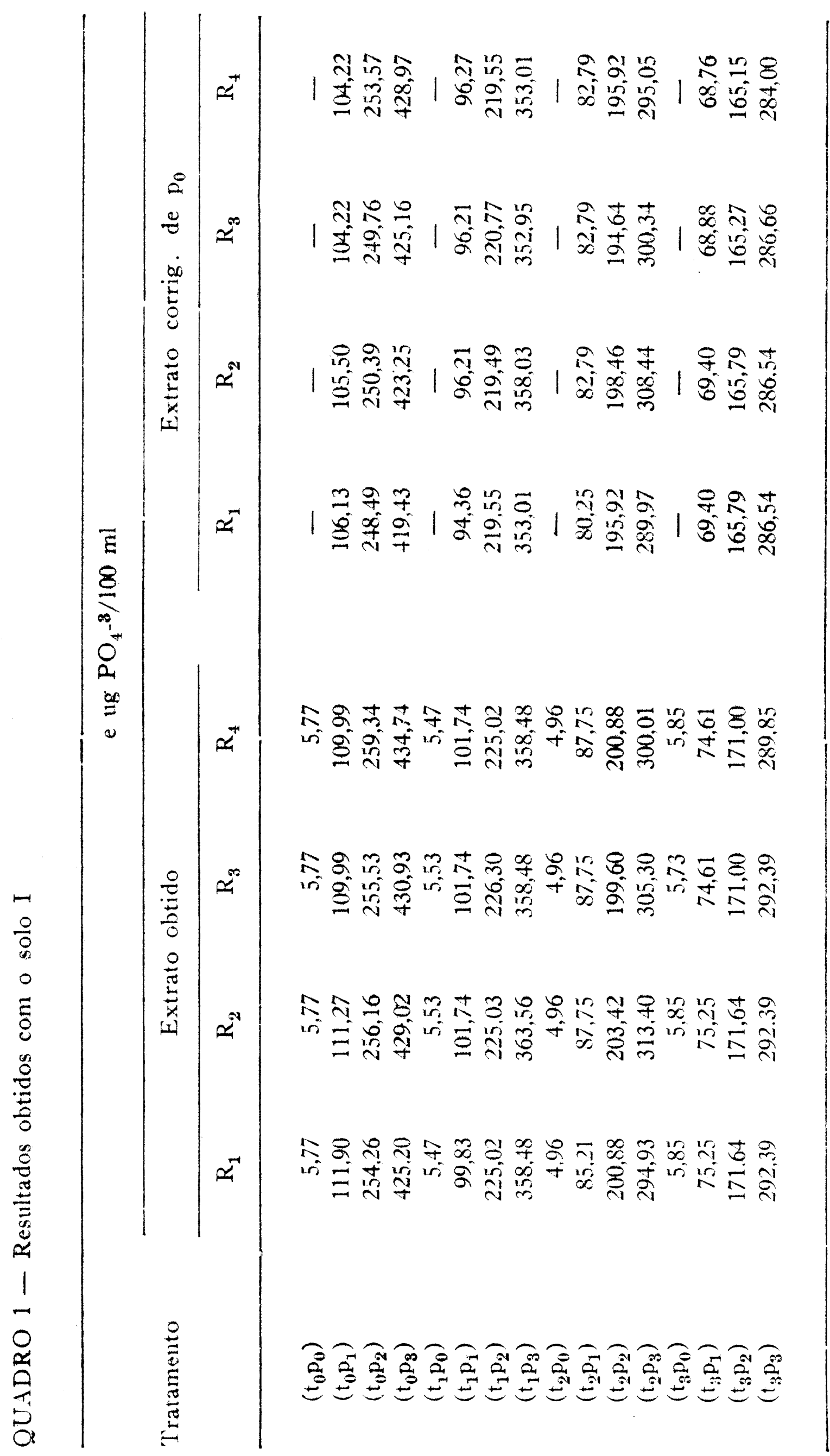




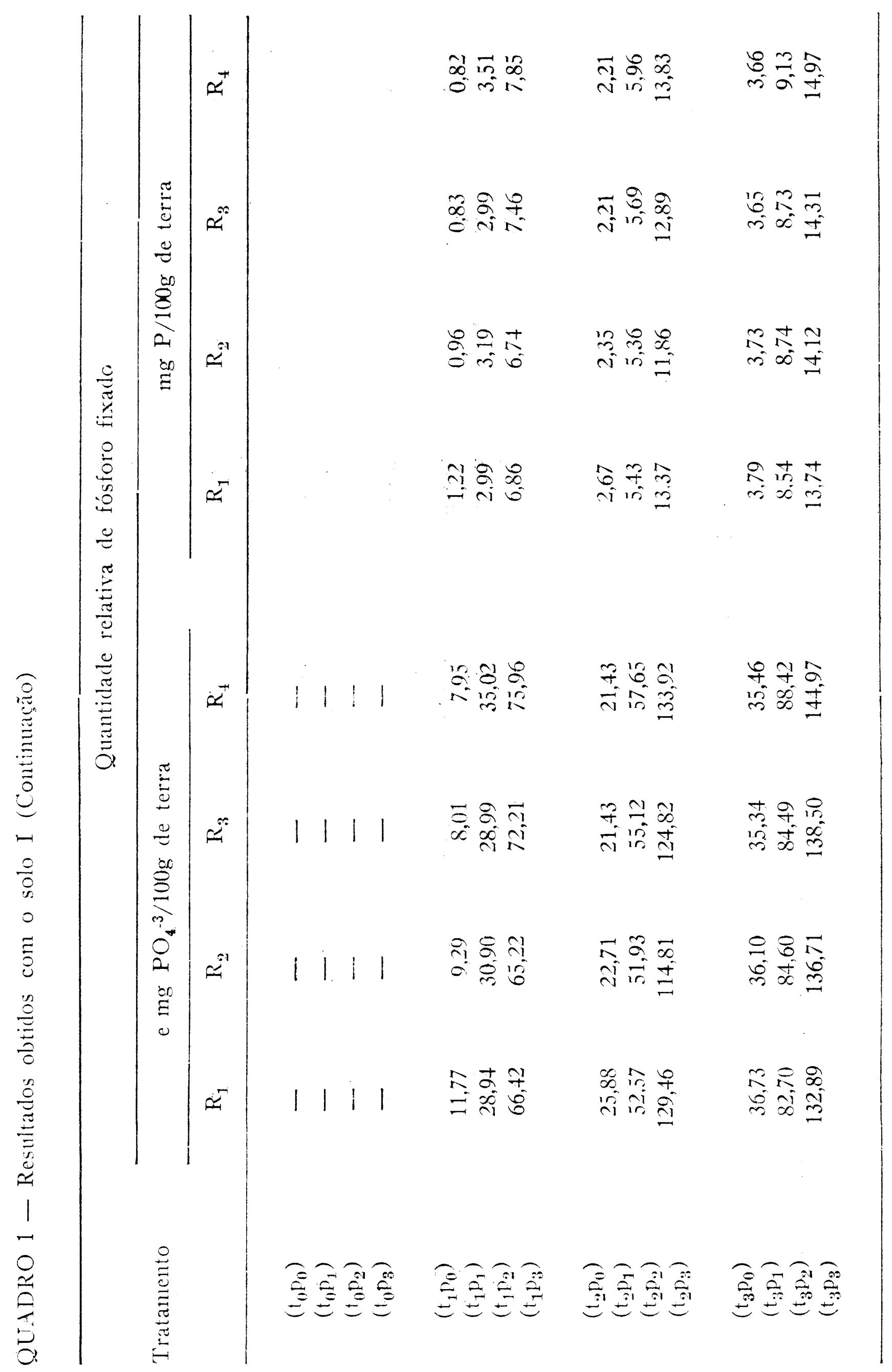


QUADRO 2 - Análise da variância

\begin{tabular}{|c|c|c|c|}
\hline Fonte de variação & GL & $Q M$ & $F^{a}$ \\
\hline 'Total & 35 & & \\
\hline Tratamentos & 8 & 87,97 & $1.659,81^{* *}$ \\
\hline Conc. da solução (P) & 2 & 20,87 & $152,28^{* *}$ \\
\hline Tempo de incubação (T) & 2 & 256,17 & $483,33^{* *}$ \\
\hline Interação T X P & 4 & 7,42 & $14,00^{* *}$ \\
\hline Ef. $L P$ em $T_{1}$ & 1 & 78,62 & $148,33^{* *}$ \\
\hline Ef. $\mathrm{L} \mathrm{P}$ em $\mathrm{T}_{2}$ & 1 & 225,89 & $462,20^{* *}$ \\
\hline Ef. $\mathrm{L} \mathrm{P}$ em $\mathrm{T}_{3}$ & 1 & 223,77 & 422,20 ** \\
\hline Ef. $Q P$ em $T_{1}$ & 1 & 2,27 & $4,28 *$ \\
\hline Ef. $Q P$ em $T_{2}$ & 1 & 11,36 & $22,43^{* *}$ \\
\hline Ef. $\widetilde{Q} P$ em $T_{3}$ & 1 & 0,12 & 0,22 \\
\hline Tempo de incubação & 2 & 80,87 & $152,58^{* *}$ \\
\hline Ef. $L$ T em $P_{1}$ & 1 & 12,26 & $23,13^{* *}$ \\
\hline Ef. L $\mathrm{T}$ em $\mathrm{P}_{2}$ & 1 & 55,30 & $104,33^{* *}$ \\
\hline Ef. $\mathrm{L} \mathrm{T} \mathrm{em} \mathrm{P}_{3}$ & 1 & 55,78 & $105,24 * *$ \\
\hline Ef. $Q \mathrm{~T}$ em $\mathrm{P}_{1}$ & 1 & 2,87 & $5,41^{*}$ \\
\hline Ef. $Q \mathrm{~T}$ em $\mathrm{P}_{2}$ & 1 & 8,12 & $15,32^{* *}$ \\
\hline Ef. $Q \mathrm{~T}$ em $\mathrm{P}_{3}$ & 1 & 57,11 & $107,75^{* *}$ \\
\hline Conc. da solução & 2 & 256,17 & $483,33 * *$ \\
\hline Resíduo & 27 & 0,53 & \\
\hline
\end{tabular}

a $*$ significativo ao nível de $5 \%$

** = significativo ao nível de $1 \%$

QUADRC 3 - Equações de regressão obtidas

\begin{tabular}{|c|c|c|c|c|c|}
\hline \multirow{2}{*}{ Efeito Considerado } & \multicolumn{3}{|c|}{ Parâmetros } & \multicolumn{2}{|c|}{ Máximo a } \\
\hline & a. & $\mathrm{b}$ & c. & $\mathrm{x}$ & Y \\
\hline Ef. $Q P$ em $T_{1}$ & 0,00000370 & $-0,001130$ & 0,6000 & & \\
\hline Ef. $Q \mathrm{P} \mathrm{cm} \mathrm{T}_{2}$ & 0,00000826 & $-0,005890$ & 3,2400 & & \\
\hline Ef. $Q P$ em $T_{3}$ & (não foi si & nificativo) & & & \\
\hline Ef. $Q T$ em $P_{1}$ & 0,00035493 & $-0,085586$ & 0,7902 & 120,56 & 5,95 \\
\hline EF. $Q$ T em $P_{2}$ & 0,00059567 & $-0,148660$ & 2,8750 & 124,78 & 12,15 \\
\hline Ef. $Q \mathrm{~T}$ em $\mathrm{P}_{3}$ & 0,00157996 & $-0,354759$ & 6,5268 & 112,26 & 26,44 \\
\hline
\end{tabular}

a - As equaçóes para o efeito quadrático de $\mathrm{P}$ em $\mathrm{T}_{1}$ e $\mathrm{T}_{2}$ não admitiram um valor máximo, para o restante dos casos. $\mathrm{X}$ é dado em horas e $\mathrm{Y}$ em $\mathrm{mg} \mathrm{P} / 100 \mathrm{~g}$ de solo. 


\section{CONCLUSÓES}

Com base na análise estatística e para as condições deste trabalho pode-se afirmar que:

a) do modo como foi obtida, a quantidade relativa de fósforo fixado presta-se muito bem para trabalhos dessa natureza;

b) os fatores estudados não agiram isoladamente;

c) não foi possível obter-se um efeito quadrático significativo para $\mathbf{P}$ dentro dos três períodos de incubação estabelecidos

d) com aproximadamente $60 \%$ do maior período de incubação seria atingido, independentemente da concentração da solução, o máximo da quantidade relativa de fósforo fixado.

STMMARY

\section{RETATIVE QUANTITY OF FIXED PHOSPHORUS IN A HYIROMORPHIC SOIL.}

Using Hydromorphic soil sample (Três Municipios Series) from the Piracicaba region, State of São Paulo, Brasil, a study was carried out to determine the phosphorus content of a solution added to the soil as compared to the quantity of fixed phosphorus. The effect of incubation period was also studied.

The relative quantity of fixed phosphorus was calculated, following a scheme previously laid down, from 4 incubation periods $(0-2-20$ and 200 hours) and 4 concentrations of a monocalcium phosphate solution $(0-500-1,000$ and $1,500 \mathrm{P} \mathrm{ppm})$.

Each treatment consisting of the equivalent of $10 \mathrm{~g}$ of oven-dry soil received $4 \mathrm{ml}$ of a solution of an already known concentration. At the end of the incubation period for each treatment, the phosphorus content of stratum was determined using an extraction solution of $0.025 \mathrm{~N} \mathrm{H}_{2}, \mathrm{SO}_{4}$ and $0,05 \mathrm{~N} \mathrm{HCl}$.

Experimental data showed that relative quantity of fixed phosphorus, under the considered conditions, fullfilled the requirements of this work. Statistical analysis of data showed that an interaction between the studied factors and that the maximum quantity of fixed phosphorus could be reached with around $60 \%$ of the longest incubation period observed.

\section{I.ITERATURA CITADA}

ANDRADF, S.S. de, 1971. Crênese e classificação de solos de três catenas nos municípios de Piracicaba e Rio Claro. Dissertação Mestrado. Piracicaba, E.S.A. "Luiz de Queiroz" - USP. 74 p. (Mimeografada). 
BASU, N.S. \& S.K. MUCHERJEE, 1972. Solubility of added phosphates in phosphate satured soil. J. Indian Soc. Soil Sci. 20(1):7-11. In Phosphorus in Agriculture Abstract n. ${ }^{\circ} 61: 57$.

CAVALCANTI, F.J. de A., 1974. Alguns aspectos da fixação do fósforo por solos da região de Piracicaba. Dissertação Mestrado. Piracicaba, E.S.A. "Luiz de Queiroz" - USP. 74 p. (Mimeografada).

MAHTAB, S.K., C.L. GOLFREY, A.R. SWOBODA \& G.W. THOMAS, 1971. Phosphorus diffusion in Soils. Soil Sci. Soc. Amer. Proc. 35(3): 393-397.

VETTORI, L., 1966. Métodos de análise de solos. Divisão de Pedologia e Fertilidade do Solo. Rio de Janeiro, 19 p. (Mimeografado).

WAUGH, D.L. \& FITTS, J.W., 1966. Estudos para interpretação de análises de solos; de laboratório e de vasos. Boletim Técnico n. ${ }^{\circ} 3$. International Soil Testing. 
